mettre à la disposition des communes, pour l'exercice du contrôle qui leur est attribué par la loi.

Frais de contrôle. - La loi du 15 juin igo6 impose au permissionnaire ou au concessionnaire les frais de contrôle: clle crée aussi une charge qui frappe explicitement l'industrie électrique, et elle s'applique à toutes les entreprises concédées ou munies de permissions de voirie, qu'elles soient antérieures ou non à la loí.

Les frais de contrôle dus à l'Etat seront perçus à forfait, conformément à un tarif arrêté chaque année par le Ministre des Travaux publics, dans les limites du maximum prévu par le décret ci-joint du 17 octobre 1907 . Les dépenses des divers services de contrôle seront réglées par décision ministérielle dans les limites du crédit inscrit à cet effet au budget de l'Etat.

Les frais de contrôle dus aux municipalités, et les dépenses nécessaires au fonctionnement des services de contrôle, sont déterminés par le Conseil municipal dans les limites prévues par le règlement d'administration publique, sans que l'Administration des Travaux publics ait à intervenir autrement que pour fournir aux services tous renseignements utiles sur les longueurs des voies canalisées.

Je vous prie de m'accuser réception de la présente circulaire. dont j'adresse directement ampliation aux ingénieurs des ponts et chaussées et des mines.

Louis Barthou.

\section{Mesure de la vitesse moyenne d'un courant d'eau}

Voici un procédé dont le principe n'est sans doute pas nouveau, mais qui a été récemment mis au point par le prol'esseur Erik ANDERSson, de Stockholm. L'application de ce procédé aux ateliers de constructions de turbines hydrauliques J. Voith, de Heidenheim (Allemagne), a fait l'objet d'une conférence faite par M. Schmitthenner à la section wurtembergeoise de la Société des Ingénieurs allem mands $\left(^{*}\right)$.

Le principe de la méthode consiste à mesurer la vitesse de déplacement d'un écran immergé dans un canal dont il occupe aussi exactement que possible toute la section transversale. Celte dernière condition limite donc l'emploi de la méthode aux canaux possédant une certaine longueur bien dressée et parfaitement régulière.

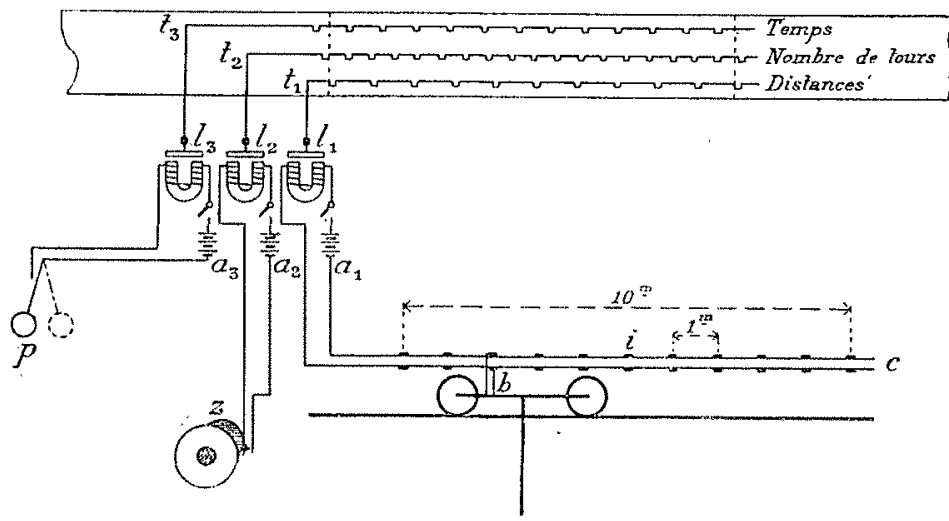

Aux Etablissements Voith, ou se trouve linstallation en question, le canal est de section rectanguiaire, avec parois au ciment lissé; il a "'2ng9g de largeur, et $2^{\text {m}} 354$ de profondeur. Sur les deux murs latéraux, on a installé des rails sur lesquels roule un chariot; celui-ci porte l'écran, cui peut ainsi se déplacer dans le canal avec un jeu de 1 centimètre. Sur une distance de 10 mètres, on a disposé, de mètre en mètre, une série de contacts $i$, disposés en dérivation sur un circuit $c$ alimenté par une batterie d'accumula-

(i) Daprès la Zeitschrift des Vercine doutsch Ingenieure. teurs $a_{i}$, et sur lequel se trouve inséré un électro aimant $l_{1}$. Sur le cadre du chariot est fixée une double lutée $b$ qui, en passant sur les contacts $i$, ferme le circuit $c$ à chaque mètie, ce qui a pour effet d'exciter l'électro $l_{1}$ et de provoquer une coche sur le diagramme de l'enregistreur.

Lorsqu'il s'agit de mesurer le rendement d'une turbine, comme c'était le cas aux Ateliers Voith, on dispose un second électro $l_{0}$, alimenté par une batterie $a_{0}$ d'accumulateurs, et établi sur un circuit fermé au passage d'une came $z$, montée sur un axe relié sans glissement avec l'axe de la turbine. Enfin, un tróisième électro $l_{3}$, relié à un pendule $p$, permet d'inscrire des longueurs proportionnelles aux temps. On a ainsi, sur un même diagramme, trois traits $t_{1}$, to et $t_{3}$, parfaitement repérés les uns par rapport aux autres, dont on peut tirer tous les éléments pour mesurer le débit instantané du cours d'eau, ainsi que le rendement du moteur hydraulique.

Pour faire une mesure, on amène le chariot un peu loin en avant, et on laisse descendre l'écran jusqu'à ce qu'il soit vertical. L'écran est alors entrainé par le courant dont il ne tarde pas à prendre la vitesse moyenne. On s'arrange pour que celte vitesse soit atteinte un peu avant la section repélée, de $10 \mathrm{~m}$. de longueur, dont il a été parlé plus haut. Un peu après cette section, on relève l'écran.

Les résultats obtenus avec ce procédé ont été estimés atteindre une grande précision. On a pu, notamment, mesurer des vitesses très faibles, de 2,5 à 5 millimètres par seconde, correspondant à des débits de 10 à 20 litres par seconde, directement jaugés à la sortie du canal. L écran et son chariot pesaient environ $40 \mathrm{kgs}$, et l'effort de traction nécessaire pour le faire avancer était de 400 grammes.

\section{Régulateur automatique de tension Pour l'Eclaimage êlectrique (")}

La lampe électrique à incandescence fait tous les jours de nouveaux progrès, et la consommation spécitique de certaines lampes s'abaisse maintenant dans des proportions importantes, néanmoins l'usage de ces lampes à faible consommatión se répand peu, en raison de leur trop grande sensibilité aux différences de potentiel variables à chaque instant sur les secteurs, et on leur préfère, malgré sa consommation beaucoup plus élevée, la lampe ordinaire de 3,5 watts par bougie, dont la durée est plus longue, à cause de la sujétion des remplacements trop fréquents des lampes, ce qui constitue un travail important dans les grands établissements, comme les théâtres par exemple, où les lampes sont en très grand nombre, et pas toujours très accessibles.

Le besoin d'un appareil pratique, permettant de règler automatiquement le voltage distribué par le secteur, se fait donc sentir tous les jours davantage, car, si d'un côté les nouvelles lampes économiques demandent, pour avoir de la durée et un bon rendement, à fonclionner avec une différence de potentiel aussi constante que possible; d'un autre côté, il devient de plus en plus difficile aux compagnies d'électricité de distribuer le courant à un voltage invariable, à cause des à-coups produits constamment par les grands consommateurs intermittents tels que : cinématographes, théâtres, enseignes lumineuses, applications qui empruntent irrégulièrement le courant à la lígnè de distribution des abonnés, à cause de leurs jeux de lumière constamment variables, etc., etc.

M. Klopfenstein a imaginé un appareil régulateur de tension ayant l'avantage de pouvoir se placer chez l'abonné, près du compteur, ou en un endroit quelconque, et ne nécessitant aucune surveillance. Cet appareil peut, paraît-il, régler le voltage à un volt près, avec une très grande régularité, quel

(") Brevet français no $36 x .446$, du 6 mai 1906 . 
que soit le nombre de lampes allumées, contrairement aux régulateurs à plots de contact, qui n'opèrent que par fractions déterminées de la résistance totale à insérer, fractions tont la valeur devrait varier avec le nombre de lampes allumées pour avóir une croissancẹ ou une décroissance régulière et constante du voltage.

Cet appareil régulateur de tension est schématiquement représenté par la figure ci-jointe.

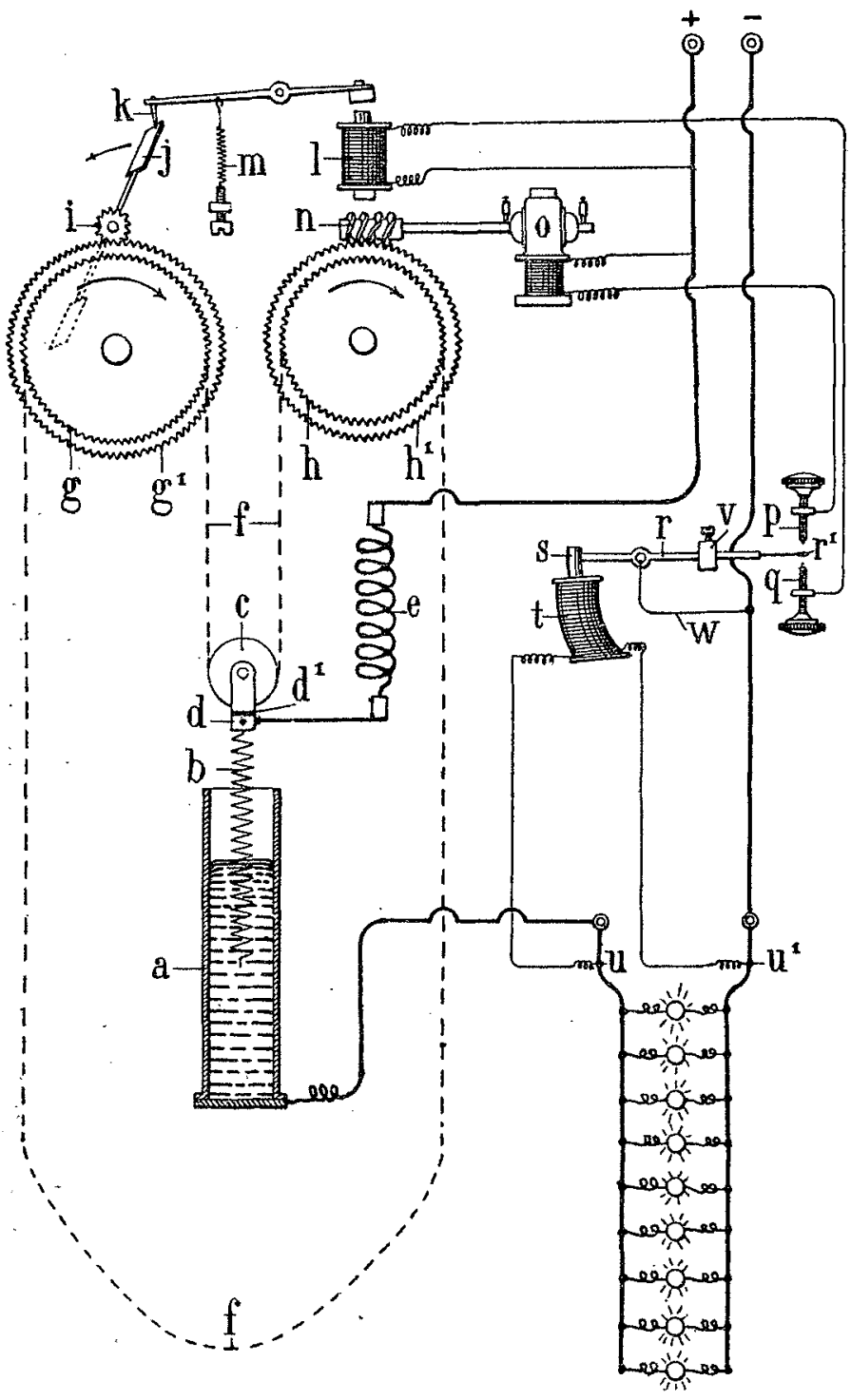

Ce régulateur automatique se compose, en principe, d'un récipient a contenant du mercure, dans lequel plonge plus ou moins une résistance $b$, suspendue à une poulie $c$ par l'intermémédiaire de la chape $d$; cette résistance $b$ est mise en communication avec le pôle + de la ligne par exemple, par l'intermédiaire d'un conducteur souple $e$ qui peut la suivre par extension ou contraction dans ses mouvements de descente et de montée; la chàpe $d$ est isolée en $d_{1}$, de manière que la totalité du courant passe par la résistance $b$. Le mercure, contenu dans le récipient $a$, est relié à l'un des pôles de l'installation.

La foulie $c$ est soutenue par une chaine $f$ passant sur les roues dentées $g, h$, solidaires respectivement des deux roues dentées $g_{1}, h_{1}$, lesquelles sont en prise avec les dispositifs commandant la descente ou la montée de la résistance $b$. La roue dentée $g$ engrène'avec un petit pignon $i$ sur l'axe duquel est monté un moulinet à ailettes J, destiné à modérer la vitesse de descente de la poulie $c$ qui l'entraine par son propre poids, lorsque ce moulinet est rendu libre par le déclanchement $\mathrm{du}$ petit doigt $k$, commandé par l'électro-aimant $l$ et le ressort de rappel $m$.

La roue dentée $h$ est commandée par une vis sans fin $n$, montêe sur l'axe d'un petit moteur électrique $o$ qui la fait tourner dans le sens indiqué par la flèche; ce mouvement a pour effet de faire remonter la poulie $c$ qui porte la résistance $b$. Lorsque le moteur o s'arrête, la poulie $c$, malgré son poids, ne peut redescendre, car le pas de la vis est assez petit pour que cette vis sans fin ne puisse être entraînée pa: la roue dentée $h$.

Le moteur $o$, et l'électro-aimant $l$, sont reliés chacun d'une manière permanente au pôls + de la ligne par une de leurs bornes, et l'atitre borne de chacun de ces appareils est reliée respectivement aux butées de contact platinées $p, q$. Ces butées de contact peuvent être touchées alternativement par l'extrémité platinée $r$ ' d'un levier $r$ dont l'autre extrémité porte un noyau de fer $s$ qui plonge dans une bobine $t$ branchée en dérivation aux deux pôles de linstallation de l'abonné, aux points $u$ et $u^{l}$, après la résistance $b$.

Ce dispositif n'est autre qu'un volimètre à contact, dont le levier $r$ est l'aiguille indicatrice. Ce levier $r$, en conséquence, s'élève ou s'abaisse suivant que le noyau de fer $s$ est plus ou moins attiré par le champ magnétique développé par la bobine $t$. Le levier $r$ porte un contrepoids curseur $v$ au mnen duquel on règle la position de la paillette platinée $r$, constituant l'extré. mité de ce levier, pour que celle-ci se trouve entre les deux vis butées $p, q$, quand le voltage est normal: i to volts par exemple

Dans ces conditions, si le voltage vient à être supérieur à t 10 volts, la bobine $t$ attire alors plus fortement le noyeau $s^{l}$, et la paillette $r$ 'vient loucher la vis butée supérieure $p$, en mettant celle-ci en communication avec le pôle - de la ligne par l'intermédiaire de l'axe d'oscillation du levier $r$ relié lui-même à ce pôle - par le fil $z$; le courant est alors transmis au moteur o qui entraine le disque $h^{l}$, ce qui a pour effet de remonter la poulie $c$, et d'augmenter la longueur de résistance $b$ întercalée entre cette poulie $c$ et le mercure contenu dans le récipient $a$. Dès qu'une certaine quantité de la résistance $b$ est intercalée dans le circuit, la différence de potentiel auxpoints $u t \iota^{1}$ retombe le levier platiné $r$ s'abaisse, abandonne par conséquent la vis butée $p$, et le moteur $o$ s'arrête.

Au conaraire, si le voltage vient à baisser, la bobine $t$ attirant moins fortement le noyau $s$, le levier $r$ est sollicité par le contrepoids 1 , la paillette $r^{l}$ s'abaisse et vient en contact avec la vis butée $q$; le courant est alors envoyé dans l'électro-aimant $l$ qui attire son armo $\alpha^{*}$. ce ce qui a pour effet de relever le doigt de retenue $k$, et de liberer le petit moulinet $j$, lequel se met en mouvement, entraîné par le poids de la poulie c. Ce mouvemont permet la descente de cette dernière, qui laisse alors plonger de plus en plus la résistance $b$ dans le mercure; la résis. tance diminuant, la différence de potentiel augmente cntre $u$ et $u^{l}$, la bobine $t$ attire le noyau $s$, le levier $r$ abandonne la vis butée $q$ et revient à sa position normale; le courant est alors rompu dans l'électro-aimant $l$, lequel abandonne son armature qui est ramenée à sa position primitive par le ressort de rappel $m$. Le doigt $k$ s'abaisse alors de nouveau, et arrête le mouvement de descente de la poulie $c$, en retenant le moulinet par une ailette.

Le présent dispositif régulateur de tension a l'avantage de pouvoir fonctionner sans aucune surveillance, il ne nécessitc que le graissage des organes de temps en temps, comme un horloge par exemple, il permet de réaliser une nolable économic sur la consommation des lampes, celle-ci n'étant plus soumises $\grave{a}$ d'aussi grandes variations de voltage et ayant par suite une durée considérablement augmentée, la variation du voltage est absolument graduelle et peut être réglée au dixième de volt si cela est nécessaire, tout dépend de la sensibilité du voltmètre à contact $t r y p q$. La résistance intercalée dans le circuit peut monter et descendre sans nécessitér l'emploi d'un moteur à renversement de marche ou tournant à contre-balais pendant la descente.

La résistance $b$ peut être maintenue au sein d'un liquide destiné à empêcher son échauffement anormal, de façon à pouvoir admettre de grandes densités de courant afin de pouvoir obtenir de grandes variations de voltage pour de trés petites variations de la longueur de la résistance $b$.

La forme, les dimensions et les détails de construction de ce système d'appareil peuvent d'ailleurs varier suivant les différentes applications. 